\title{
Neural Coupling Binds Visual Tokens to Moving Stimuli
}

\author{
Michael Rose and Christian Büchel \\ NeuroImage Nord, Department for Systems Neuroscience, University Medical Center Hamburg-Eppendorf, D-20246 Hamburg, Germany
}

Spatially separated visual objects that appear in alternating sequence can be perceived as a single moving object. This phenomenon of apparent motion enables us to perceive sequentially presented images as a motion picture. How does the visual system bind together single visual tokens to one moving object? Here we report a series of experiments investigating apparent motion with electroencephalographic recordings showing that gamma-band oscillatory coupling in the visual cortex is crucial for this phenomenon. We used an ambiguous stimulus that could be perceived as moving either vertically or horizontally. Because visual information from the right and left visual hemifields is routed to the contralateral early visual cortex, in the case of perceived horizontal movement, information from both hemispheres has to be integrated. In accord with this assumption, our data show stronger oscillatory coupling between right and left visual cortices during perception of horizontal motion compared with vertical motion.

Key words: perception; gamma band; binding; EEG; coherence; neural coupling

\section{Introduction}

Synchronization of oscillatory electrical brain activity in the gamma band $(\sim 40 \mathrm{~Hz})$ has been proposed as a basic mechanism underlying transient functional coupling of neural assemblies (von der Malsburg, 1994). Many studies in the visual system of the cat (Gray et al., 1989; Engel et al., 1991) and monkey (Bressler et al., 1993; Kreiter and Singer, 1996; Maldonado et al., 2000) have lent support to this hypothesis. Apart from increases in oscillatory coupling during associative learning (Miltner et al., 1999), there is increasing evidence that object features represented by spatially distinct neural assemblies are dynamically linked to a coherent percept by synchronized activity in the gamma range (Engel et al., 2001). This can be simple bar stimuli that move coherently through the receptive fields of two neurons (Engel and Singer, 2001), different images presented to each eye as in binocular rivalry (Srinivasan et al., 1999; Fries et al., 2002), or complex visual stimuli such as faces (Rodriguez et al., 1999; Tallon-Baudry and Bertrand, 1999).

The processing of apparent motion posits a similar binding problem as object recognition: at least two visual objects segregated in time and space need to be perceptually linked to represent a single moving stimulus (Korte, 1915). Binding by synchrony is one possible neuronal mechanism underlying apparent-motion perception (Knyazeva et al., 1999).

At the level of the primary visual cortex, visual information from each visual hemifield (right and left) are separated and almost exclusively routed to the contralateral hemisphere (Palmer,

Received April 6, 2005; revised Aug. 18, 2005; accepted Sept. 9, 2005.

This work is supported by grants from the Deutsche Forschungsgemeinschaft and the Bundesministerium für Bildung und Forschung. C.B. and M.R. are supported by the Volkswagenstiftung. We thank K. Friston, B. Röder, A. Engel, L. Ungerleider, and A. Kleinschmidt for comments on a previous draft of this paper.

Correspondence should be addressed to Dr. Michael Rose, Neurolmage Nord, Department of Systems Neuroscience, University Medical Center Hamburg, Martinistrasse 52, D-20246 Hamburg, Germany. E-mail: rose@uke.uni-hamburg.de.

DOl:10.1523/JNEUROSCI.2998-05.2005

Copyright $\odot 2005$ Society for Neuroscience 0270-6474/05/2510101-04\$15.00/0
1999). Thus, an apparent-motion stimulus that comprises two visual tokens presented alternating in the left and in the right hemifield are perceived as moving horizontally only if visual information from the left and right hemispheres are integrated (Chaudhuri et al., 1991).

To test the hypothesis that synchronous oscillatory coupling between visual cortices is linked to apparent-motion perception in humans, we used an ambiguous apparent-motion stimulus that has two equally valid alternative perceptual interpretations (i.e., horizontal or vertical movement). These two clearly separable percepts allow a straightforward prediction, namely that if motion is perceived horizontally, oscillatory coupling should increase between hemispheres (Chaudhuri and Glaser, 1991). Coupling is investigated by electroencephalogram (EEG) recordings.

In the principal experiment, an ambiguous apparent-motion stimulus was presented, in which the movement of two visual tokens (dots) can be perceived as moving either horizontally or vertically (Chaudhuri and Glaser, 1991) (see Fig. 1). This stimulus consists of two sets of two diagonally opposite dots that are flashed in alternation. In the first experiment, we experimentally manipulated the stimulus percept by slowly $\left(0.08^{\circ} / \mathrm{s}\right)$ decreasing the horizontal distance between dots until the percept changed to horizontal movement. In the second experiment, we manipulated the perceptual change by obliterating the upper or left tokens, respectively. In a third experiment, the stimulus was held constant and the participants were free to switch between the two perceptual states. In all experiments, volunteers indicated a change of their percept by a button press.

\section{Materials and Methods}

Volunteers and stimuli. Three experiments were conducted using an ambiguous apparent-motion stimulus (Fig. 1). A total of 40 healthy volunteers with normal or corrected-to-normal vision participated in the experiments (Fig. 1). The probability of whether this stimulus is perceived as either moving horizontally or vertically depends on the distance between the dots (Hock et al., 1993). In an equidistant arrangement, there is a bias toward vertical motion perception. This bias can be avoided by 

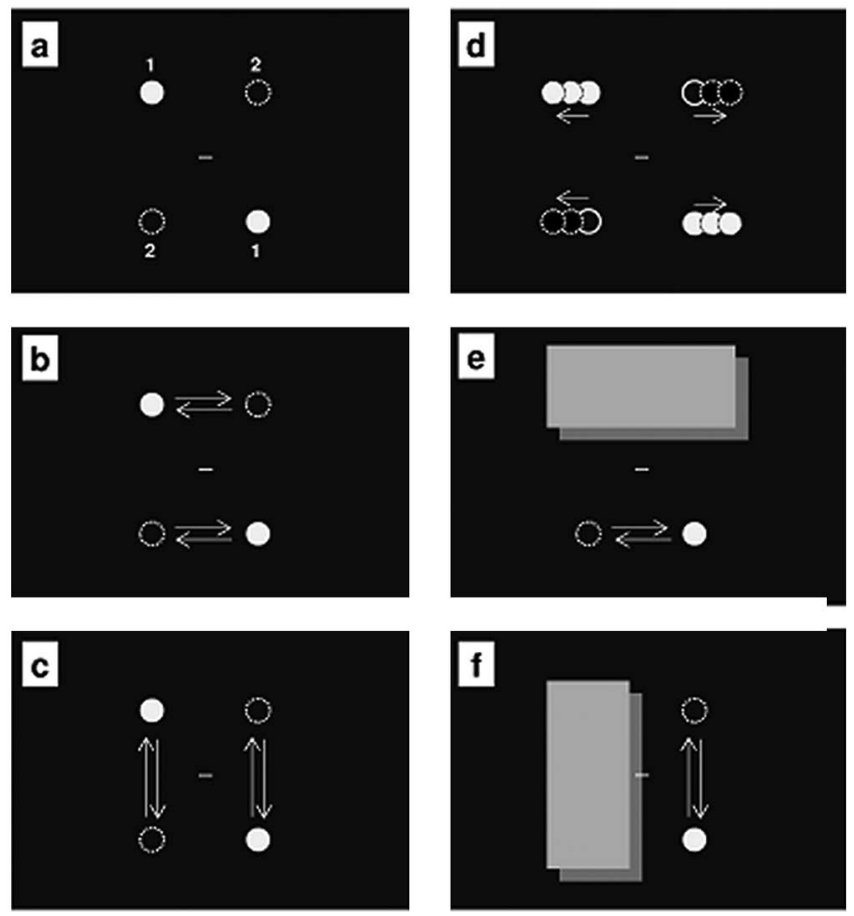

Figure 1. Apparent-motion stimulus layout. $\boldsymbol{a}$, Two low-contrast diagonal tokens (filled gray circles; diameter: $0.85^{\circ}, 2.7 \mathrm{~cd} / \mathrm{m}^{2}$ ) appeared at position 1 for $200 \mathrm{~ms}$ and were then replaced by the tokens at position 2, presented for $200 \mathrm{~ms}$. The background was black $(0.05$ $\left.\mathrm{cd} / \mathrm{m}^{2}\right)$. Subjects kept their gaze at the fixation spot in the middle throughout each experimental run. The fixation dot was either a small vertical or horizontal line representing the current percept. This stimulus can be perceived as moving either horizontally $(\boldsymbol{b})$ or vertically $(\boldsymbol{c})$. The horizontal and vertical distance between dots from center to center was $6.9^{\circ}$.d, In experiment 1 , the distance between horizontal dots was decreased by $0.08^{\circ}$ for each dot presentation. $\boldsymbol{e}, \boldsymbol{f}$, In experiment 2 , perception was biased toward horizontal $(\boldsymbol{e})$ or vertical $(\boldsymbol{f})$ apparent motion by temporarily $(750 \mathrm{~ms}$, every $10 \mathrm{~s}$ ) obliterating the upper or left tokens, respectively. To keep the horizontal percept more stable, we used a constant horizontal distance of the tokens of $6.2^{\circ}$ (aspect ratio, 1:1.11). In experiment 3, volunteers viewed the stimulus and reported spontaneous perceptual switches by button presses. To avoid a bias toward vertical movement, as has been observed in equidistant (i.e., quadratic) stimulus displays (Chaudhuri and Glaser, 1991), the horizontal distance between tokens was set to $5.1^{\circ}$ (aspect ratio, 1:1.34).

using a horizontal-to-vertical-distance ratio of 1/1.3 (i.e., shorter horizontal distance) (Chaudhuri and Glaser, 1991). In experiment 1 ( $n=12$; mean age, 27 years; range, $24-30$; six females), perceptual switches were induced by changing the horizontal distance between the dots (Fig. 1d). In experiment 2 ( $n=11$; mean age, 28 years; range, 23-40; three females), perceptual switches were induced by occluding either the left or top row of dots. In experiment $3(n=17$; mean age, 25 years; range, 24-40; eight females), the stimulus display was kept constant throughout the presentation.

Stimuli were presented using a personal computer that ensured synchronization with the EEG recording unit using the software Presentations. A liquid crystal display monitor with a constant-refresh rate of 60 $\mathrm{Hz}$ was used for stimulus presentation, with a viewing distance of $1 \mathrm{~m}$. Participants signaled perceptual switches by pressing a button in experiments 1 and 3, and indicated a loss of induced percept in experiment 2 . Furthermore, the subjects had the option to press a second button in cases in which neither of the two motions was perceived. The study was approved by the local ethics committee, and all subjects gave written informed consent before inclusion into this study.

Data acquisition. An EEG was recorded from 34 channels (BrainVision system amplifier; BrainProducts, Munich, Germany) (impedance, $<5$ $\mathrm{k} \Omega$; low cutoff, $0.03 \mathrm{~Hz}$; high cutoff, $250 \mathrm{~Hz} ; 500 \mathrm{~Hz}$ sampling rate referred to the nose). Vertical and horizontal electro-oculograms were recorded from above versus below the left eye and from the outer canthi of the eyes, respectively.
Data analysis. Data segments of 2000 ms were extracted starting 1000 ms after an indicated perceptual switch. Epochs with artifacts caused by blinks or eye movements (vertical and horizontal), amplifier saturation, and fast amplitude shifts $(>100 \mu \mathrm{V}$ within $2 \mathrm{~ms})$ were excluded from additional analysis. For each subject, the minimal number of epochs for the horizontal or vertical condition was computed (mean of 21 epochs in experiment 1; mean of 30 epochs in experiment 2; mean of 20 epochs in experiment 3 ) and was used for additional analysis of both conditions within that subject. Therefore, for each subject, the number of data segments was the same for both conditions to avoid problems with the sample size bias. The epochs were decomposed using a wavelet-based time-frequency analysis (Tallon-Baudry et al., 1998) (C. Torrence and G. Compo, http://paos.Colorado.EDU/research/wavelets/). For each trial, the signal was convolved with complex Morlet's wavelets (wave number $=6$ ), with $f_{0}$ ranging from 1 to $120 \mathrm{~Hz}$ in $1 \mathrm{~Hz}$ steps. The wavelet basis was normalized to have a total energy of 1 at all scales. Wavelet-based coherence was estimated for each subject and separately for the horizontal and vertical percept. The mean coherence spectra were calculated across a time series of $2000 \mathrm{~ms}$ and were $Z$-transformed (Jarvis and Mitra, 2001).

Coherence was computed using the cross-spectral density $(x p)$ of channel $k$ and channel $m$, and the power spectral densities $p k$ and $p m$ in the time $(t)$-frequency $(f)$ domain as follows:

$$
C_{t, f}=\frac{\left|x p_{t, f}\right|^{2}}{p m_{t, f} p k_{t, f}} .
$$

The transformed spectra were averaged and then transformed back to obtain the grand mean coherence spectra (see Fig. 2). Single-subject $Z$-transformed coherence values were extracted in the frequency range between 30 and $50 \mathrm{~Hz}$ and were compared between horizontal and vertical percepts. Frequencies $<10 \mathrm{~Hz}$ were not investigated because of the likely confound of stimulus-related low-frequency oscillatory activity (visual tokens alternated every $200 \mathrm{~ms}$ ).

Phase locking $(P L)$ between the two EEG channels $k$ and $m$ was computed in the time-frequency domain using the phase $(p)$ of the signal for each trial $(j)$, and then averaged across trials $(n)$ (Tallon-Baudry et al., 2001; Gruber and Muller, 2005) as follows:

$$
P L=\left|\frac{1}{n} \sum_{j=1}^{n} e^{i\left(p_{j, k}(t, f)-\left(p_{j, m}(t, f)\right.\right.}\right| .
$$

\section{Results}

According to our hypothesis, we investigated coherence differences between horizontal and vertical motion percepts. We found increasing coherence between hemispheres for the horizontal percept compared with the vertical percept, with a maximum over extrastriate visual cortex (electrodes P7/P8). This effect was observed when perceptual changes were induced by (1) varying the distance of the dots (experiment 1; paired $t$ test for electrode pair P7/P8, $t_{(11)}=2.4 ; p<0.05$ ) and by (2) occluding parts of the stimulus (experiment 2; paired $t$ test for electrode pair $\left.\mathrm{P} 7 / \mathrm{P} 8, t_{(10)}=2.9 ; p<0.05\right)$.

However, one might argue that, in experiment 1, physical stimulus properties were not absolutely identical, and the occlusion of dots in experiment 2 might represent a confound for ensuing changes in percept. We therefore conducted experiment 3 , in which the perceptual switches were intrinsically "generated" by the participants without any stimulus alteration. In this experiment, we observed a significant increase in coherence in the frequency band between 30 and $50 \mathrm{~Hz}$, between left and right extrastriate visual electrodes during the perception of horizontal motion (ANOVA with factors electrode pair: O1-O2, PO7-PO8, P7-P8; and Percept: vertical, horizontal; main effect Percept, $\left.F_{(1,16)}=11.9 ; p<0.05\right)$. The interaction effect of electrode by percept $\left(F_{(2,32)}=10.1 ; p<0.05\right)$ demonstrated that the maximal 
Exp. 2

Conerence

Exp. 3
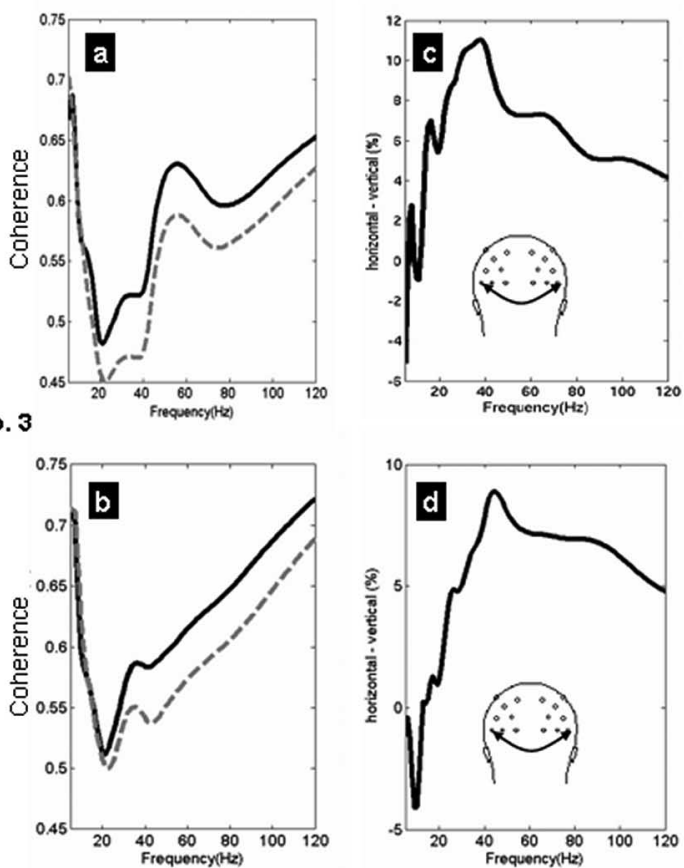

Spectral Power Difference
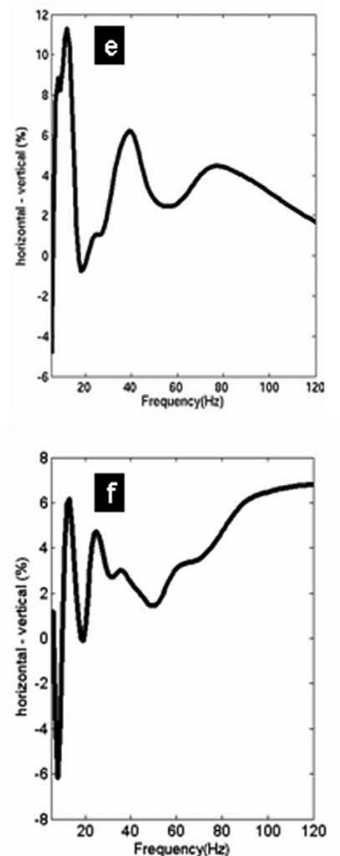

Figure 2. Increased coupling across hemispheres during horizontal motion perception. Coherence estimates $(\boldsymbol{a}, \boldsymbol{b})$ for the horizontal (solid line) and vertical (dashed line) percept are plotted separately for experiment 2 (percept change induced by occluding dots) and experiment 3 (constant stimulus). Grand mean coherence was calculated by combining the Z-transformed single-subject coherence spectra and transforming the $Z$ values back to obtain coherence. The coherence differences for the horizontal minus the vertical percept are plotted in percentage as a function of frequency $(c, d)$ and show a peak of $\sim 40 \mathrm{~Hz}$ for both experiments. Plots $\boldsymbol{e}$ and $\boldsymbol{f}$ show spectral power differences for the horizontal minus the vertical percept in percentage, indicating no significant difference in relation to the percept compared across both experiments.
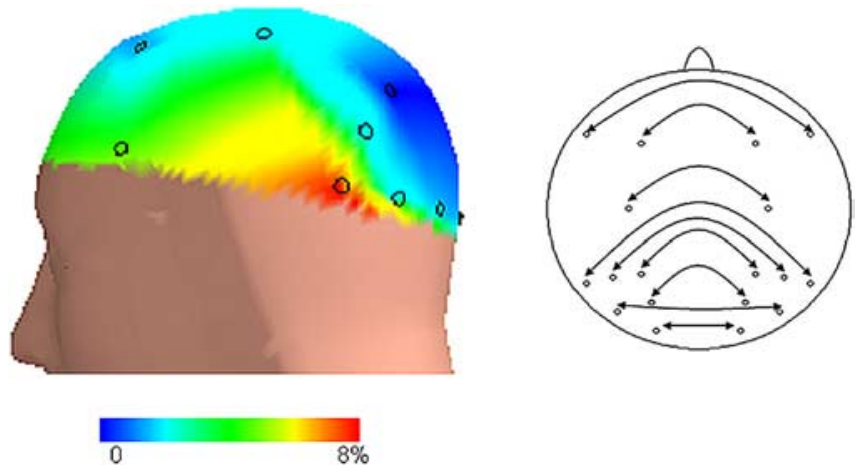

Figure 3. Specificity of coherence differences. We estimated coherence for right-left pairs of electrodes and show the scalp distribution of these coherence differences between vertical and horizontal percepts. It is clearly visible that the largest difference was observed over extrastriate visual cortex sites.

difference to the vertical condition $(\sim 9 \%)$ was observed again at electrode pair P7-P8, (paired $t$ test between horizontal and vertical percept for this electrode, $t_{(16)}=3.9 ; p<0.05$ ). Additional inspection of different frequency bands revealed that the coherence differences peaked between 30 and $50 \mathrm{~Hz}$ (Fig. 2).

In addition, we estimated the coherence between right-left symmetric pairs of electrodes to further investigate the topographical maximum of the effect. Again, this analysis revealed that the maximal change in coherence for horizontal versus vertical percept was observed at lateral extrastriate visual electrode sites (P7/P8) (Fig. 3).

For experiment 3 , the power spectra were also analyzed for

electrodes P7/P8 and for the horizontal and vertical percept. The corresponding ANOVA (factors, electrode and percept) showed no difference in the power of the analyzed band $(30-50 \mathrm{~Hz})\left[F_{(1,16)}=0.2\right.$ (not significant); mean $\pm \mathrm{SE}$, vertical, $110 \pm 23 \mu \mathrm{V}^{2}$; horizontal, $\left.114 \pm 29 \mu \mathrm{V}^{2}\right]$.

\section{Discussion}

Our data clearly show an increased oscillatory coupling between hemispheres when perceiving horizontal compared with vertical apparent motion and provide the first evidence for the importance of oscillatory coupling in apparent-motion perception. Importantly, this result was obtained while volunteers were viewing a physically identical stimulus and only the percept changed. This extends previous reports, highlighting the relevance of oscillatory coupling of high-frequency components for binocular rivalry (Fries et al., 2002) and face perception (Rodriguez et al., 1999).

Previous EEG and magnetoencephalography studies investigating apparent motion have reported changes in $\alpha$ (Basar-Eroglu et al., 1996; Struber and Herrmann, 2002) and gamma power (Basar-Eroglu et al., 1996) at individual electrodes immediately preceding perceptual switches. Thus, it is important to investigate whether evoked gamma activity also differed during stable percepts, which could trivially explain increased coherence between hemispheres. However, this was not the case. Gamma power in the analyzed data segments was identical for horizontal and vertical motion percepts; thus, the observed coupling between hemispheres cannot be explained by a simple increase in gamma power during the perception of horizontal apparent motion. Furthermore, phase locking between electrodes, a measure that is independent of amplitude differences (Varela et al., 2001), revealed a statistically significant increase in the gamma band during horizontal motion perception between hemispheres (P7-P8; $\left.t_{(16)}=2.8 ; p<0.05\right)$ and thus confirmed the coherence estimates.

The peak of the oscillatory gamma band coupling around electrodes P7-P8 (Fig. 3), which are close to the medial temporal area (MT) (supplemental Fig. 1, available at www.jneurosci.org as supplemental material), adds to existing evidence that extrastriate visual cortex, including human area MT (hMT), is involved in apparent-motion perception. This notion is in accordance with functional magnetic resonance imaging studies showing increased activity in area hMT when a visual stimulus was perceived as moving compared with a percept of flickering dots (Goebel et al., 1998; Sterzer et al., 2003). MT neurons have receptive fields that cross the vertical meridian, a property that allows them to integrate visual information across both visual hemifields, as would be necessary to perceive horizontal apparent motion in the stimulus used (Maunsell et al., 1987). A possible anatomical basis has been revealed by anatomical labeling studies, showing that neurons in MT are directly connected to contralateral MT (Maunsell and Van Essen, 1987).

When using EEG to measure coupling between different brain 
areas, volume conduction of the head leads to a high absolute coherence estimate, especially between neighboring electrodes. Furthermore, absolute coherence estimates can also depend on the used reference electrode. It is important to note that, in all of our paradigms, the vertical percept can be regarded as a rigorous baseline that controls for both effects. Consequently, we report only the difference of neuronal coupling in relation to different percepts of apparent motion. It is unlikely that volume conduction or the reference electrode can change as a function of percept, and therefore, the observed difference in coherence is related to the perceptual difference.

The probabilities for the vertical and the horizontal percepts were found to be equal if the horizontal distance between dots was smaller (Chaudhuri and Glaser, 1991). Interestingly, this bias for vertical motion perception vanishes when the whole stimulus is presented within a single visual hemifield (Chaudhuri and Glaser, 1991). This suggests the involvement of transcallosal neurotransmission in the observed perceptual bias, which is supported by animal studies showing that synchronous activity in the gamma band across the hemispheres ceased when the corpus callosum was transected (Engel et al., 1991; Munk et al., 1995; Kiper et al., 1999). Although potentially more difficult to assess, split-brain patients are impaired in horizontal apparent-motion perception (Gazzaniga, 1987), especially in the context of low stimulus contrast (Naikar, 1996), as used in our task.

In an important theoretical framework for consciousness, binding by synchrony was hypothesized to play a role in the formation and competition of neural ensembles, especially when there is perceptual competition such as in ambiguous displays (Crick and Koch, 2003). This notion is strongly supported by our data showing that oscillatory coupling depends on perceptual awareness in an ambiguous stimulus setting in which competition between percepts occurs (Chaudhuri and Glaser, 1991).

\section{References}

Basar-Eroglu C, Struber D, Kruse P, Basar E, Stadler M (1996) Frontal gamma-band enhancement during multistable visual perception. Int J Psychophysiol 24:113-125.

Bressler SL, Coppola R, Nakamura R (1993) Episodic multiregional cortical coherence at multiple frequencies during visual task-performance. Nature 366:153-156.

Chaudhuri A, Glaser DA (1991) Metastable motion anisotropy. Vis Neurosci 7:397-407.

Crick F, Koch C (2003) A framework for consciousness. Nat Neurosci 6:119-126.

Engel AK, Singer W (2001) Temporal binding and the neural correlates of sensory awareness. Trends Cogn Sci 5:16-25.

Engel AK, Konig P, Kreiter AK, Singer W (1991) Interhemispheric synchronization of oscillatory neuronal responses in cat visual-cortex. Science 252:1177-1179.

Fries P, Schroder JH, Roelfsema PR, Singer W, Engel AK (2002) Oscillatory neuronal synchronization in primary visual cortex as a correlate of stimulus selection. J Neurosci 22:3739-3754.

Gazzaniga MS (1987) Perceptual and attentional processes following callosal section in humans. Neuropsychologia 25:119-133.

Goebel R, Khorram-Sefat D, Muckli L, Hacker H, Singer W (1998) The constructive nature of vision: direct evidence from functional magnetic resonance imaging studies of apparent motion and motion imagery. Eur J Neurosci 10:1563-1573.
Gray CM, Konig P, Engel AK, Singer W (1989) Oscillatory responses in cat visual-cortex exhibit inter-columnar synchronization which reflects global stimulus properties. Nature 338:334-337.

Gruber T, Muller MM (2005) Oscillatory brain activity dissociates between associative stimulus content in a repetition priming task in the human EEG. Cereb Cortex 15:109-116.

Hock HS, Kelso JA, Schoner G (1993) Bistability and hysteresis in the organization of apparent motion patterns. J Exp Psychol Hum Percept Perform 19:63-80.

Jarvis MR, Mitra PP (2001) Sampling properties of the spectrum and coherency of sequences of action potentials. Neural Comput 13:717-749.

Kiper DC, Knyazeva MG, Tettoni L, Innocenti GM (1999) Visual stimulusdependent changes in interhemispheric EEG coherence in ferrets. J Neurophysiol 82:3082-3094.

Knyazeva MG, Kiper DC, Vildavski VY, Despland PA, Maeder-Ingvar M, Innocenti GM (1999) Visual stimulus-dependent changes in interhemispheric EEG coherence in humans. J Neurophysiol 82:3095-3107.

Korte A (1915) Kinematoskopische untersuchungen. Z Psychol 72:194-296.

Kreiter AK, Singer W (1996) Stimulus-dependent synchronization of neuronal responses in the visual cortex of the awake macaque monkey. J Neurosci 16:2381-2396.

Maldonado PE, Friedman-Hill S, Gray CM (2000) Dynamics of striate cortical activity in the alert macaque: II. Fast time scale synchronization. Cereb Cortex 10:1117-1131.

Maunsell JH, Van Essen DC (1987) Topographic organization of the middle temporal visual area in the macaque monkey: representational biases and the relationship to callosal connections and myeloarchitectonic boundaries. J Comp Neurol 266:535-555.

Miltner WH, Braun C, Arnold M, Witte H, Taub E (1999) Coherence of gamma-band EEG activity as a basis for associative learning. Nature 397:434-436.

Munk MH, Nowak LG, Nelson JI, Bullier J (1995) Structural basis of cortical synchronization. II. Effects of cortical lesions. J Neurophysiol 74:2401-2414.

Naikar N (1996) Perception of apparent motion of colored stimuli after commissurotomy. Neuropsychologia 34:1041-1049.

Palmer SE (1999) Vision Science. Cambridge, MA: MIT.

Rodriguez E, George N, Lachaux JP, Martinerie J, Renault B, Varela FJ (1999) Perception's shadow: long-distance synchronization of human brain activity. Nature 397:430-433.

Srinivasan R, Russell DP, Edelman GM, Tononi G (1999) Increased synchronization of neuromagnetic responses during conscious perception. J Neurosci 19:5435-5448.

Sterzer P, Eger E, Kleinschmidt A (2003) Responses of extrastriate cortex to switching perception of ambiguous visual motion stimuli. NeuroReport 14:2337-2341.

Struber D, Herrmann CS (2002) MEG alpha activity decrease reflects destabilization of multistable percepts. Brain Res Cogn Brain Res 14:370-382.

Tallon-Baudry C, Bertrand O (1999) Oscillatory gamma activity in humans and its role in object representation. Trends Cogn Sci 3:151-162.

Tallon-Baudry C, Bertrand O, Peronnet F, Pernier J (1998) Induced gamma-band activity during the delay of a visual short-term memory task in humans. J Neurosci 18:4244-4254.

Tallon-Baudry C, Bertrand O, Fischer C (2001) Oscillatory synchrony between human extrastriate areas during visual short-term memory maintenance. J Neurosci 21:RC177(1-5).

Varela F, Lachaux JP, Rodriguez E, Martinerie J (2001) The brainweb: phase synchronization and large-scale integration. Nat Rev Neurosci 2:229-239.

von der Malsburg C (1994) The correlation theory of brain function. In: Models of neural networks II (Schulten K, ed), pp 95-119. Berlin: Springer. 